\title{
A Fed Back Level-Set Method for Moving Material-Void Interfaces
}

\author{
Barry Koren and Arthur Venis
}

\begin{abstract}
This paper is a feasibility study of a level-set method for the computation of moving material-void interfaces in an Eulerian formulation. The paper briefly introduces level-set methods and focuses on the development of such a method, that does not just accurately resolve the geometry of the interface, but also the physical quantities at and near the interface. Results are presented for illustrative model problems. As concerns its ability to improve the geometrical resolution of free boundaries, as expected, the level-set method performs excellently. Concerning the improvement of physical (all other than merely geometrical) free-boundary properties, the method performs very well for downstream-facing fronts and is promising for upstream-facing ones.
\end{abstract}

\section{Introduction}

For the computation of free-boundary flows on a fixed grid (Eulerian approaches), since many years, some well-proven techniques exist. Known examples of these are the marker-and-cell (MAC) method [1] and the volume-of-fluid (VOF) method [2]. An interesting method arisen last decade is the level-set method. A text book on level-set methods is [6], a classical journal paper is [4].

The accurate capturing of moving fronts on fixed grids is not trivial because of the non-smoothness of the fronts. No advantage can be taken of nice numerical accuracy properties, valid for smooth solutions only. As a natural fix to this, in the level-set method, to the system of physical unknowns, an additional, non-physical unknown is added: the level-set function, say $\phi$, which is smooth at the front. Furthermore, an additional, non-physical equation is added: a convection equation for $\phi$. The level-set function has a pre-defined, unique and constant value at the interface. To accurately resolve the material interface, one can best keep track of this pre-defined interface value for $\phi$, say $\phi_{f}$. The easy possibility for creating smoothness at the interface (through an artificial, passive scalar function) is a first interesting property of the level-set method. Related to this, a second interesting property is that the interface's location is neatly defined (viz., as the location where $\phi=\phi_{f}$ ). With a physical jump at the interface (e.g., a density jump) from $c=1$ to $c=0$, in case of a diffused grid-representation of this jump, it is not immediately clear how to precisely define the interface location. (Should one define it as there 
where $c=\frac{1}{2}$ or $c=h$, with $h$ the mesh size, or as whatever?) A third interesting property of the level-set technique is that the level-set equation requires no new, specifically tailored discretization method; the discretization method that one has in mind for the physical system, can be easily and consistently extended with the equation for $\phi$. As opposed to fluid markers or volume-of-fluid fractions, the levelset function can be directly embedded in the already existing, discrete system of physical equations. Related to this, a fourth advantage of the level-set method is that there is no difficulty in extending it from 2-D to 3-D.

When one is not only interested in an accurate geometrical resolution of the interface, but also in an improved resolution of physical quantities at and near the interface, the level-set approach still offers a possibility that seems to be new. A physically sound feedback of the level-set function may be incorporated into the real (physical) equations. In the level-set literature known to us, if there is a feedback of the level-set function $\phi$ into physics, it is restricted to material properties such as the ratio of specific heats $(\gamma=\gamma(\phi))$ and the kinematic viscosity $(\nu=\nu(\phi))$. In the present level-set method, the level-set function will be explicitly fed back into the computation of the physical flux function $f=f(c)$. I.e., in the discrete case, we extend this to $f=f(c, \phi)$.

\section{Test cases and reference results}

In the model problems to be considered, the convection of circular and square interfaces is the issue. The problems are described by the convection equation

$$
\frac{\partial c}{\partial t}+u \frac{\partial c}{\partial x}+v \frac{\partial c}{\partial y}=0, \quad(x, y) \in[-1,1] \times[-1,1],
$$

with as initial conditions: the "circle"

$c(x, y, t=0)= \begin{cases}1, & (x, y) \in\left(x-x_{c}\right)^{2}+\left(y-y_{c}\right)^{2} \leq\left(\frac{1}{5}\right)^{2}, x_{c}=y_{c}=-\frac{1}{2}, \\ 0, & \text { elsewhere, }\end{cases}$

and the "square"

$$
c(x, y, t=0)= \begin{cases}1, & (x, y) \in\left[x_{c}-\frac{1}{5}, y_{c}-\frac{1}{5}\right] \times\left[x_{c}+\frac{1}{5}, y_{c}+\frac{1}{5}\right] \\ & x_{c}=y_{c}=-\frac{1}{2} . \\ 0, & \text { elsewhere }\end{cases}
$$

For the velocity field, defined for positive $c$ only (i.e., in the material only), we simply take

$$
u=v=1,
$$

and for the inlet boundary conditions

$$
c(x=-1, y, t)=c(x, y=-1, t)=0 .
$$

Requested for both the circle and the square: $c(x, y, t=1)$. The exact solutions are identical to the initial solutions, (2a) and (2b), but now with $x_{c}=y_{c}=\frac{1}{2}$. 

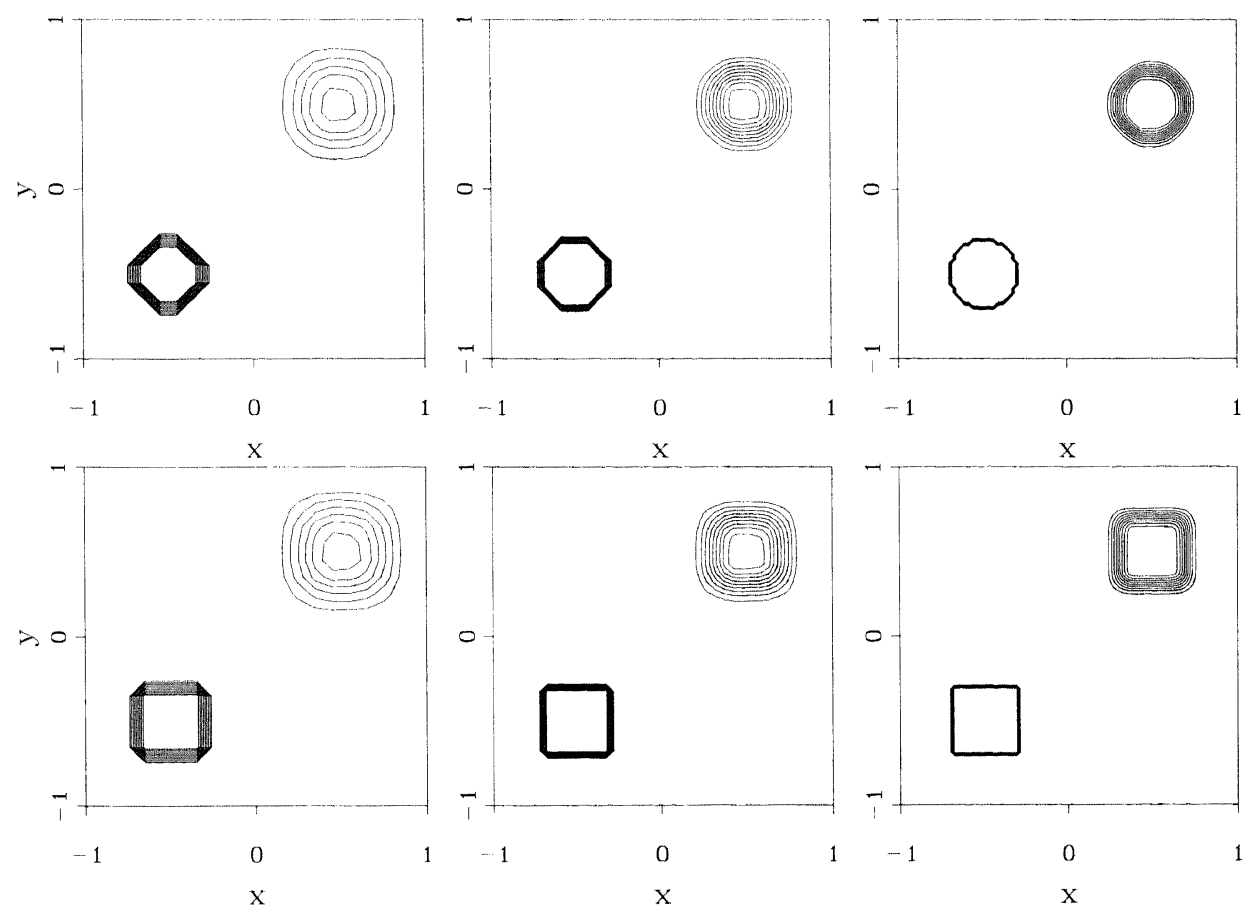

Figure 1. Initial and final numerical solutions for convection circle (up) and square (down) with standard numerical scheme (limited $\kappa=\frac{1}{3}$ ), for from left to right: $h=\frac{1}{10}, \frac{1}{20}, \frac{1}{40}$.

As grids, we apply finite-volume grids with successively $20 \times 20,40 \times 40$ and $80 \times 80$ cells. Applying for the space discretization a cell-centered, limited $\kappa=\frac{1}{3}$ scheme [3] (which is formally third-order accurate in monotonous solution parts as well as at smooth extrema), and for the time integration an explicit four-stage Runge-Kutta scheme, we obtain the results given in Figure 1. In the lower left corners of all six graphs, the corresponding discrete initial solutions have been depicted. (Iso-lines are given at $c=0.1 n, n=1, \ldots, 9$.)

\section{Simple level-set method: no feedback with physics}

\subsection{Principle}

To explain the level-set method - first without feedback - consider the 1-D convection equation

$$
\frac{\partial c}{\partial t}+u \frac{\partial c}{\partial x}=0, \quad u=\text { constant }>0
$$




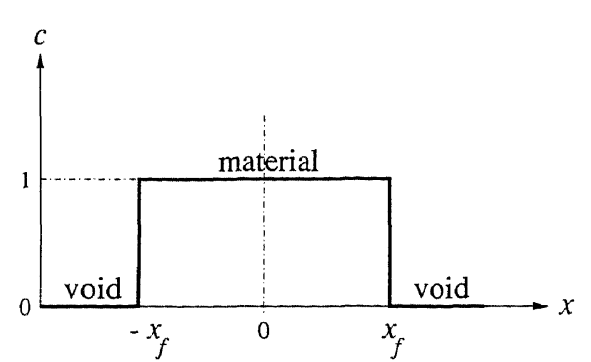

a. Physical function.

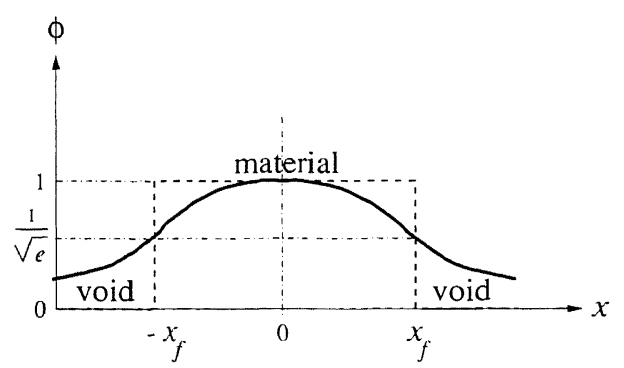

b. Level-set function.

FIGURE 2. Initial solutions.

With the level-set function $\phi$, the extended equation reads

$$
\frac{\partial q}{\partial t}+u \frac{\partial q}{\partial x}=0, \quad q=\left(\begin{array}{c}
c \\
\phi
\end{array}\right), \quad u=\text { constant }>0 .
$$

Suppose that the initial solution $c(x, t=0)$ looks as in the sketch in Figure 2a, so with interfaces at $x= \pm x_{f}$ and with jumps from $c=1$ to $c=0$ over there. Then, for the corresponding initial distribution of the level-set function, $\phi(x, t=0)$, we propose the probability curve

$$
\phi(x, t=0)=e^{-\frac{1}{2}\left(\frac{x}{x_{f}}\right)^{2}} .
$$

A sketch of (7) is given in Figure $2 \mathrm{~b}$. Note that (7) is infinitely many times differentiable at all points (including $x=0$, a linear level-set function would not be differentiable there). Higher-order accurate convection schemes can take full advantage of this. Further note that the function has been chosen such that its inflection points (its maximum slopes) coincide with the interfaces. (This gives the best posedness of the interface-detection problem.) We still remark that the choice (7) for the level-set function is rather arbitrary. Other functions, with equally good differentiation properties, and interface values $\phi_{f}$ different from $\frac{1}{\sqrt{e}}$, could have been chosen. For a multi-D problem, e.g., the 2-D problem of which the initial material-void interface is sketched in Figure 3, the choice of the initial level-set function may be done in the following way. Take some point in the material and define that as the origin of a local $r, \theta$-coordinate system (Figure 3 ). Next take as the initial level-set function

$$
\phi(r, \theta, t=0)=e^{-\frac{1}{2}\left(\frac{r}{r_{f}}\right)^{2}}
$$

where $r_{f}=r_{f}(\theta)$ is the radial distance from the point chosen to the material interface, for a given angle $\theta, \theta \in[0,2 \pi]$. Doing so, we have $\phi_{f}=\frac{1}{\sqrt{e}}$ all over the interface. For many shapes, this initialization works, also in $3-\mathrm{D}$, where it carries over in a spherical coordinate system. 


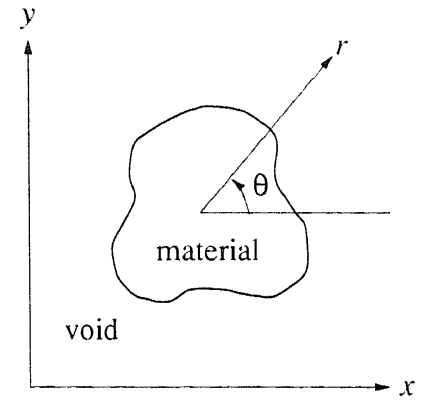

FIGURE 3. 2-D material-void interface.

\subsection{Velocity field}

A subtle property of the convection of material-void interfaces is that the velocity field is only defined in the material. Hence, for the convection of the level-set function in the void region, an artificial velocity field still has to be defined. The opportunity to make this choice, without being inhibited by physics, is a good chance in fact to improve the free boundary's resolution. E.g., in the void region an artificial velocity can be chosen which counteracts the effects of numerical diffusion of the physical quantity $c$. For the 1-D convection equation (5), in the void region a velocity may be defined which looks as sketched in Figures $4 \mathrm{a}$ and $4 \mathrm{~b}$. So, for a downstream-facing front, $u \frac{\partial \phi}{\partial x}<0$, at the void side of the interface, a velocity may be chosen which is smaller than the velocity at the material side of the interface (Figure 4a). This artificial anti-convection implies converging characteristics and may thus lead to re-steepening of a diffused front. To realize the steepening in case of an upstream-facing front, $u \frac{\partial \phi}{\partial x}>0$, the void velocity has to be taken greater than the material velocity (Figure $4 \mathrm{~b}$ ).

Of course, a difficulty here is to not affect too seriously the physically correct propagation speed of the front. Therefore, in this paper we do not apply anticonvection. Our present suggestion for the choice of the artificial velocity field in the void region is to take that as the smoothest possible extrapolation of the velocity field at the material interface.

\subsection{Results}

For the two test cases defined in Section 2, we can already present practically relevant level-set results now, viz. for the convection of the shape.

For comparison, we consider the corresponding, exact discrete solutions and the standard numerical (limited $\kappa=\frac{1}{3}$ ) results. For both the exact discrete solution and the solution obtained by the standard approach, the material interface is defined as the iso-line $c_{f}=h$ ( $h$ being the mesh width). In the standard approach, as mentioned in Section 1, just a proper definition of the material interface is not 


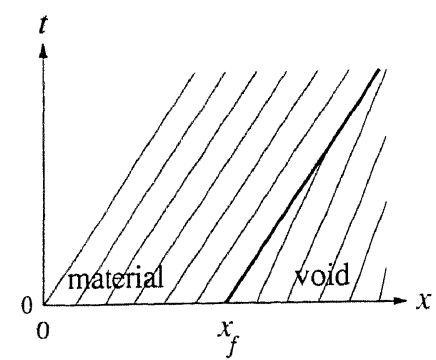

a. Downstream-facing interface.

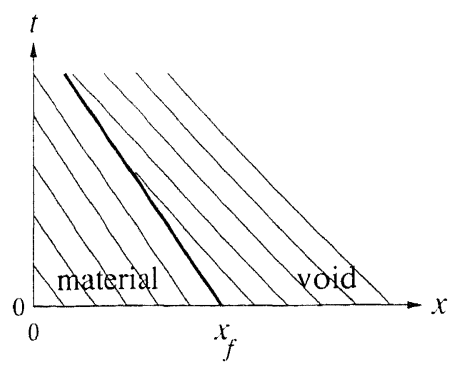

b. Upstream-facing interface.

FIGURE 4. Anti-convection for moving material-void interfaces.

trivial already. In the level-set approach, this definition already exists: the isoline $\phi_{f} \equiv \frac{1}{\sqrt{e}}$. For the $80 \times 80$-grid, in Figure 5, we present the discrete shapes at $t=1$, from left to right: (i) according to the exact discrete solution $c$, (ii) according to the limited numerical solution $c$ and (iii) according to the (nonlimited) numerical solution $\phi$. (The iso-lines in the two middle graphs belong to the same solutions already depicted in the two most right graphs in Figure 1.) For the circle, the difference in quality between the standard numerical results and the level-set results is striking. Seen from the graph, the shape preservation of the level-set method is very good; numerical errors are very small. The depicted level-set circle is even more accurate than the plotted, exact discrete circle. The quality of the latter suffers from the interface-definition problem. For the square, the level-set solution is less accurate than for the circle (because of the loss of one order of accuracy at the corners). Nevertheless, here as well, the level-set result is still much better than the standard numerical result.

\section{Extended level-set method: feedback with physics}

As mentioned, the useful knowledge to be extracted from the numerical solution of the level-set function does not need to be restricted to geometrical improvement of the free boundary only. Knowledge obtained from the level-set solution can also be fed back into the discretization of the physical equations. This coupling of the level-set function to physics is application-dependent and much effort may be put into it. We proceed by briefly presenting one such coupling (more couplings are given in [3]). The model equation to be considered still is

$$
\frac{\partial q}{\partial t}+u \frac{\partial q}{\partial x}+v \frac{\partial q}{\partial y}=0, \quad q=\left(\begin{array}{c}
c \\
\phi
\end{array}\right), \quad u=v=1 .
$$

An obvious physical argument is that in the downstream void region, the physical fluxes (i.e., the $c$-fluxes) must be zero. Besides a front itself, its upstream and downstream sides can also be distinguished by means of the level-set function. 

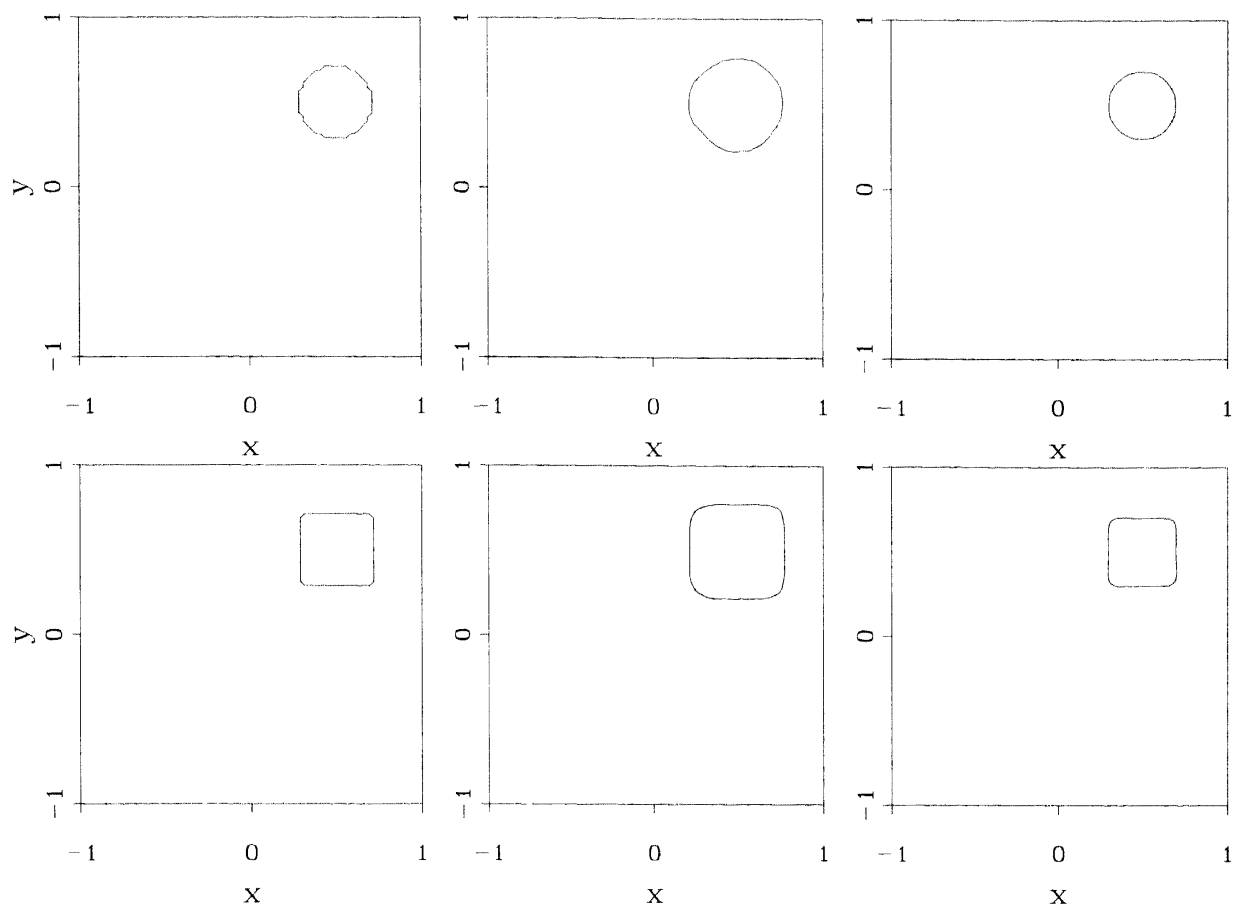

Figure 5. Final shapes for circle (up) and square (down), for $h=\frac{1}{40}$ and for, from left to right: exact discrete solution $c$ (isoline $c=c_{f}=h$ ), limited $\kappa=\frac{1}{3}$ solution $c$ (iso-line $c=c_{f}=h$ ) and non-limited $\kappa=\frac{1}{3}$ solution $\phi$ (iso-line $\phi=\phi_{f}=\frac{1}{\sqrt{e}}$ ).

E.g., in 2-D, the downstream void region is there where

$$
(u, v) \cdot \nabla \phi<0 \text { and } \phi<\phi_{f},
$$

and the upstream void region is there where

$$
(u, v) \cdot \nabla \phi>0 \text { and } \phi<\phi_{f} \text {. }
$$

The required cell-face gradient $\nabla \phi$ can be evaluated in a standard second-order accurate way. Then, the algorithm reads as follows. In the downstream void region, all fluxes are put to zero. In the upstream void region, this closing of cell faces does not work [3]. There, we apply an appropriate compressive limiter (superbee, [5]) for counteracting diffusion of the material interface. In the material itself we apply the limited $\kappa=\frac{1}{3}$ discretization.

In Figure 6, numerical results are presented for this level-set approach with coupling, for both the convection of the circle and the square. Particularly good results appear to be obtained at the downstream-facing parts of the interfaces. 

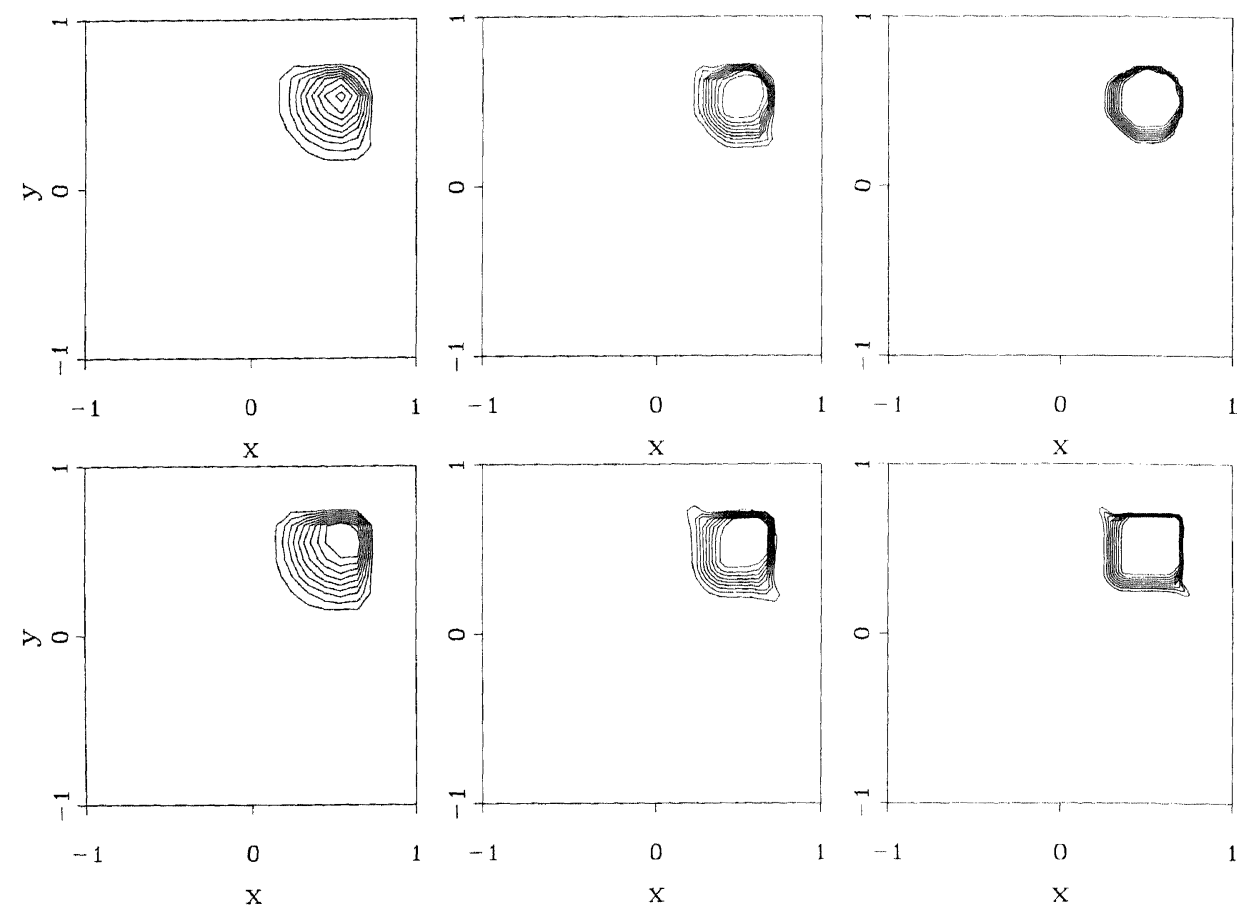

FIgURE 6. Numerical solutions $c$ for convection circle (up) and square (down) with fed back level-set scheme, for from left to right: $h=\frac{1}{10}, \frac{1}{20}, \frac{1}{40}$.

We illustrate this once more by means of the following simplifications of the two model problems. The equation and velocity to be considered are still the same, but the initial solutions differ. Instead of (2a), first we consider a downstream-facing curvilinear front only, viz.

$c(x, y, t=0)= \begin{cases}1, & (x, y) \in\left(x-x_{c}\right)^{2}+\left(y-y_{c}\right)^{2} \leq\left(\frac{1}{2}\right)^{2}, x_{c}=y_{c}=-1, \\ 0, & \text { elsewhere, }\end{cases}$

and likewise, instead of (2b), the downstream-facing rectilinear front

$$
c(x, y, t=0)= \begin{cases}1, & (x, y) \in\left[x_{c}, y_{c}\right] \times\left[x_{c}+\frac{1}{2}, y_{c}+\frac{1}{2}\right], x_{c}=y_{c}=-1 . \\ 0, & \text { elsewhere. }\end{cases}
$$

The appropriate boundary conditions are given in [3].

In Figure 7, the numerical solutions $c$ at $t=1$ are given, as obtained with the standard (limited $\kappa=\frac{1}{3}$ ) scheme. In Figure 8, the corresponding results obtained with the fed-back level-set approach are shown. Close observation learns that the effect of closure of cell faces in the void region does not only lead to a thinning of 

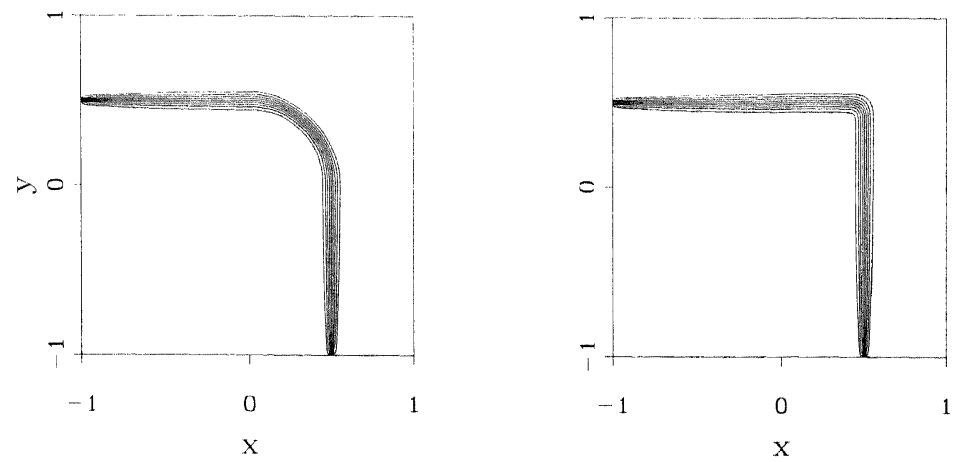

Figure 7. Curvilinear (left) and rectilinear (right) numerical solutions $c$ for convection downstream-facing interfaces, obtained through standard (limited $\left.\kappa=\frac{1}{3}\right)$ scheme $\left(h=\frac{1}{40}\right)$.
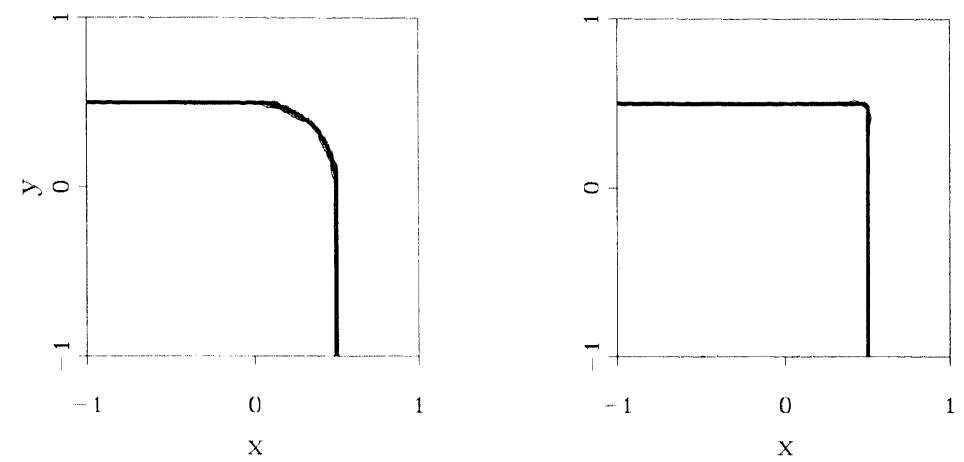

Figure 8. Curvilinear (left) and rectilinear (right) numerical solutions $c$ for convection downstream-facing interfaces, obtained through fed back level-set scheme $\left(h=\frac{1}{40}\right)$.

the interface part diffused into the void region, but also to a thinning of the part diffused into the material.

\section{Conclusions}

A challenging possibility of level-set methods, presented in this paper, is to convect the level-set function as an active (instead of as a passive) scalar. (For shapetracking purposes only, as expected, the level-set approach without feedback yields excellent results.) When feeding information about the level-set function back into the discretization of the physical equations, for downstream-facing material-void 
interfaces strongly improved physical results can be obtained. For upstream-facing fronts, the fed back level-set method is still open for further improvement.

\section{References}

[1] F.H. Harlow and J.E. Welch, Numerical calculation of time-dependent viscous incompressible flow of fluid with free surfaces, Phys. Fluids, 8 (1965) 2182-2189.

[2] C.W. Hirt and B.D. Nicholls, Volume of fluid (VOF) method for dynamics of free boundaries, J. Comput. Phys., 39 (1981) 201-225.

[3] B. Koren and A.C.J. Venis, A level-set method for moving material-void interfaces, Report MAS-R9731 (1997) CWI, Amsterdam.

Also: http://www.cwi.nl/static/publications/reports/MAS-1997.html

[4] S. Osher and J.A. Sethian, Fronts propagating with curvature-dependent speed: algorithms based on Hamilton-Jacobi formulations, J. Comput. Phys., 79 (1988) 12-49.

[5] P.L. Roe, Some contributions to the modelling of discontinuous flows, Large-Scale Computations in Fluid Mechanics, Lectures in Applied Mathematics, 22, Part 2, 163-193 (1985) American Mathematical Society, Providence, Rhode Island.

[6] J.A. Sethian, Level Set Methods: Evolving Interfaces in Geometry, Fluid Mechanics, Computer Vision, and Materials Science (1996) Cambridge University Press, Cambridge, U.K.

CWI,

P.O. Box 94079,

1090 GB Amsterdam, The Netherlands

E-mail address: Barry.Koren@cwi.nl

MacNeal-Schwendler (E.D.C.) B.V.,

Groningenweg 6,

2803 PV Gouda, The Netherlands

E-mail address: Arthur.Venis@master.macsch.com 